\title{
Multiple Oscillatory Modes of the Global Ocean Circulation
}

\author{
Wilbert Weijer* and Henk A. DijKstra \\ Institute for Marine and Atmospheric Research Utrecht, Department of Physics and Astronomy, Utrecht University, Utrecht, Netherlands
}

(Manuscript received 31 October 2002, in final form 6 May 2003)

\begin{abstract}
For the first time, the (linear) stability of the global ocean circulation has been determined explicitly. In a low-resolution general circulation model, a steady state is computed directly by solving the elliptic boundary value problem. The stability of this solution is determined by solving the generalized eigenvalue problem. Although the steady global circulation is (linearly) stable, there are two interesting oscillatory modes among the least stable ones, with periods of about 3800 and 2300 yr. These modes are characterized by buoyancy anomalies that propagate through the ocean basins as they are advected by the global overturning circulation. The millennial timescale is set by the time it takes for anomalies to travel, at depth, from the North Atlantic to the North Pacific. Further analyses confirm that the advective feedback between the steady flow and buoyancy anomalies is an essential process in the propagation mechanism. The growth rate of the millennial modes is controlled by vertical mixing. It is argued that these internal ocean modes may be a relevant mechanism for global climate variability on millennial timescales.
\end{abstract}

\section{Introduction}

More and more evidence on past variability of the climate system suggests that distinct millennial timescale variations have occurred. This so-called sub-Milankovitch variability can be found in ocean sediment and ice-core data (Bond et al. 1997; Mayewski et al. 1997), and seems to pervade the entire late Pleistocene (Oppo et al. 1998). Bond et al. (1997) have argued for a pervasive 1-2-kyr oscillation in the climate system, which is the origin of the Dansgaard-Oeschger cycles during glacial times and of the less strong variations in the Holocene, such as the Medieval Warm Period and the Little Ice Age.

The mechanism underlying this climatic rhythm is still under debate. There are some indications that the Holocene part of the $1.5-\mathrm{kyr}$ cycle is linked to variability in solar irradiance (Bond et al. 2001). Hence, the variability could be forced by a periodic component of the external forcing. Linear amplification mechanisms within the climate system should then operate to amplify the weak signal into a noticeable and recordable climatic

* Current affiliation: Scripps Institution of Oceanography, La Jolla, California.

Corresponding author address: Wilbert Weijer, Physical Oceanography Research Department, Scripps Institution of Oceanography, La Jolla, CA 92093-0230.

E-mail: wweijer@ucsd.edu signal. However, it seems unlikely that this weak source of variability in external forcing is sufficient to explain the large-amplitude response of the climate system associated with the Dansgaard-Oeschger cycles; the internal nonlinear dynamics of the climate system is likely to be important, with a dominant role for the ocean's thermohaline circulation (Broecker 2000).

Most of the hypotheses to explain the DansgaardOeschger cycles gather around two possibilities; the first ascribes this climate rhythm to switches between equilibria or "excitable states" of the thermohaline circulation (e.g., Broecker et al. 1985; Weaver and Hughes 1994; Ganopolski et al. 2001). These switches may be excited by stochastic components in the forcing (coherence resonance), in which case the transitions are irregularly paced. However, when the noise is assisted by a weak periodic component (stochastic resonance: Alley et al. 2001; Ganopolski and Rahmstorf 2002), then the pacing between the transitions may become more regular, as observed for the Dansgaard-Oeschger cycles.

An alternative explanation is advocated by Sakai and Peltier (1995, 1996, 1997, 1999), who suggest that the variability on millennial timescales is generated by internal ocean modes. Indeed, (damped) oscillatory motion can already be found in the simplest models to capture characteristics of thermohaline circulation and, in fact, two of these prototype oscillations seem to have counterparts in more realistic models of the ocean circulation. First there is Welander's (1982) so-called flip- 
flop oscillator, which features two boxes that are stacked vertically. The water in these boxes mix when the density of the upper layer exceeds that of the lower layer. The density difference is determined by the competing effects of salinity and temperature. This model contains oscillations that are characterized by a slow and diffusive buildup of potential energy that is suddenly released in a catastrophic overturning episode. A similar mechanism is responsible for the so-called deep-decoupling oscillations or flushes that are found in many primitive equation models of the thermohaline circulation (e.g., Marotzke 1989; Winton and Sarachik 1993). The oscillations are generally in the millennial range.

The other type of oscillation is generally referred to as loop or overturning oscillation (Welander 1986). It is characterized by the advection of (mainly) salt anomalies by the basic circulation, modifying the overturning strength in an oscillatory fashion. In the classical two-box model of Stommel (1961), two reservoirs are coupled through pipes and exchange fluid at rates proportional to the density differences between the boxes. Regions in parameter space exist where both a thermally driven and a salinity driven solution can be stable. Stommel (1961) shows that the thermally driven solution is a stable node but that the salinity driven solution is a stable spiral, thus attracting trajectories in an oscillatory fashion. Although Dewar and Huang (1995) exclude the possibility that the model of Stommel (1961) contains self-sustained oscillations, spontaneous instabilities do occur in related versions of this model: for instance, the four-box model of Tziperman et al. (1994), Welander's (1986) loop oscillator, and double-hemispheric box models (Scott et al. 1999; Titz et al. 2002) (albeit as an unstable limit cycle). The time scale of this type of oscillation depends on the basin size and is generally in the centennial range for Atlantic-size basins.

The overturning oscillation seems to have counterparts in primitive-equation models, as well. It is found in two- and three-dimensional models of single-hemispheric flows (Winton and Sarachik 1993), and in latitude-depth models of double-hemispheric thermohaline circulation (Mysak et al. 1993; Quon and Ghil 1995; Schmidt and Mysak 1996; Sakai and Peltier 1995; Dijkstra and Molemaker 1997). Sakai and Peltier (1996) extent their work on double-hemispheric flows by coupling three latitude-depth models in the south through a circumpolar channel as a representation of the global ocean. They find small-amplitude overturning oscillations with centennial timescales, and show that the period increases when freshwater is added to the North Atlantic. When this freshwater input exceeds a certain threshold, the dominant mode of variability changes into large-amplitude deep-decoupling oscillations with millennial timescales. This behavior is consistent with their results of a single-basin model (Sakai and Peltier 1995), and is robust when the ocean model is coupled to an atmospheric energy balance model (Sakai and Peltier 1997).

In a fully three-dimensional global context, however, this mode of variability seems harder to find. Although the 320-yr oscillation found in the Hamburg large-scale geostrophic (LSG) model by Mikolajewicz and MaierReimer (1990) stands out as the best known example of an overturning oscillation in a three-dimensional global model, Pierce et al. (1995) and Osborn (1997), in fact, argue that this oscillation is rather an expression of Welander's flip-flop oscillator active in the Southern Ocean. Yet, Sirkes and Tziperman (2001) were able to identify a damped oscillatory mode using the adjoint of the Geophysical Fluid Dynamics Laboratory (GFDL) climate model. They claim that this mode may be responsible for interdecadal variability in the North Atlantic. However, the mode lacks the three-dimensional pattern that is characteristic for (inter-)decadal oscillations in a single-hemispheric basin (e.g., Te Raa and Dijkstra 2002). The 530-yr period of the oscillatory mode suggests that it may be an overturning mode.

In this paper, we report on the explicit identification of multiple internal ocean modes of the global ocean circulation within a low-resolution ocean general circulation model. These modes are detected by investigating the (linear) stability of the global ocean circulation. Systematic investigations of the stability and variability of the large-scale ocean circulation have been performed before, for instance in two-dimensional models of the thermohaline circulation (Schmidt and Mysak 1996; Vellinga 1996; Dijkstra and Molemaker 1997) and in simplified three-dimensional geometries (Huck and Vallis 2001; Weijer and Dijkstra 2001). Sirkes and Tziperman (2001) used an adjoint model of the GFDL model to assess the stability of the global circulation. But never before has the complete eigenvalue problem been solved explicitly for the fully three-dimensional global ocean circulation.

It is shown that in this model internal oscillations with typical millennial timescales appear as damped modes. The focus in the paper is on the physical mechanisms of propagation of the anomalies and the characterization of the eigenvalues in terms of energy production and decay. In section 2, the model configuration is outlined, whereas in section 3 a the basic steady solution is presented. A description of its most dangerous oscillatory modes is found in section $3 \mathrm{~b}$, and the physical mechanism is discussed in section 3c. Section 4 discusses the results and concludes this paper.

\section{The global implicit ocean model}

The model that is used in this study has been described in Weijer et al. (2003). Here the basic equations and parameterizations are shortly revisited. The model here extends that in Dijkstra et al. (2001) by applying the equations below to a global configuration with the inclusion of realistic bathymetry, wind forcing, and ther- 
mohaline forcing. The ocean velocities in eastward and northward directions are indicated by $u$ and $v$, the vertical velocity by $w$, the pressure by $p$, and the temperature and salinity by $T$ and $S$. Vertical and horizontal mixing of heat and salt are represented by eddy diffu- sivities with (constant) horizontal and vertical diffusivities $\kappa_{h}$ and $\kappa_{v}$ for heat. The mixing coefficients of salt are taken equal to those of heat.

The governing equations are

$$
\begin{aligned}
\frac{D u}{d t}-\frac{u v \tan \phi}{r_{0}}-2 \Omega_{0} v \sin \phi & =-\frac{1}{\rho_{0} r_{0} \cos \phi} \frac{\partial p}{\partial \lambda}+Q_{\tau}^{\lambda}+\mathcal{F}^{u}, \\
\frac{D v}{d t}+\frac{u^{2} \tan \phi}{r_{0}}+2 \Omega_{0} u \sin \phi & =-\frac{1}{\rho_{0} r_{0}} \frac{\partial p}{\partial \phi}+Q_{\tau}^{\phi}+\mathcal{F}^{v}, \\
\frac{\partial p}{\partial z} & =-\rho g, \\
0 & =\frac{\partial w}{\partial z}+\frac{1}{r_{0} \cos \phi}\left[\frac{\partial u}{\partial \lambda}+\frac{\partial(v \cos \phi)}{\partial \phi}\right], \\
\frac{D T}{d t} & =\kappa_{h} \nabla_{h}^{2} T+\kappa_{v} \frac{\partial^{2} T}{\partial z^{2}}+Q_{T}, \\
\frac{D S}{d t} & =\kappa_{h} \nabla_{h}^{2} S+\kappa_{v} \frac{\partial^{2} S}{\partial z^{2}}+Q_{S}, \text { and } \\
\rho & =\rho(T, S),
\end{aligned}
$$

with

$$
\begin{aligned}
\frac{D F}{d t} & =\frac{\partial F}{\partial t}+\frac{u}{r_{0} \cos \phi} \frac{\partial F}{\partial \lambda}+\frac{v}{r_{0}} \frac{\partial F}{\partial \phi}+w \frac{\partial F}{\partial z}, \\
\boldsymbol{\nabla}_{h} F & =\left(\frac{1}{r_{0} \cos \phi} \frac{\partial F}{\partial \lambda}, \frac{1}{r_{0}} \frac{\partial F}{\partial \phi}\right), \text { and } \\
\boldsymbol{\nabla}_{h} \cdot \mathbf{F} & =\frac{1}{r_{0} \cos \phi} \frac{\partial}{\partial \lambda} F^{\lambda}+\frac{1}{r_{0} \cos \phi} \frac{\partial}{\partial \phi}\left(F^{\phi} \cos \phi\right),
\end{aligned}
$$

where $F$ and $\mathbf{F}=\left(F^{\lambda}, F^{\phi}\right)$ are an arbitrary scalar and vector, respectively.

The ocean circulation is driven by a wind stress $\tau(\lambda, \phi)=\tau_{0}\left(\tau^{\lambda}, \tau^{\phi}\right)$, where $\tau_{0}$ is the amplitude and where $\tau^{\lambda}(\lambda, \phi)$ and $\tau^{\phi}(\lambda, \phi)$ provide the spatial patterns. These patterns are constructed by interpolating the Trenberth et al. (1989) wind stress data onto the model grid. As in many low-resolution ocean general circulation models, the surface forcing is distributed as a body forcing over a certain depth of the upper ocean using a vertical profile function $g(z)$ :

$$
Q_{\tau}^{\lambda}=g(z) \frac{\tau_{0}}{\rho_{0} H_{m}} \tau^{\lambda} \text { and } \quad Q_{\tau}^{\phi}=g(z) \frac{\tau_{0}}{\rho_{0} H_{m}} \tau^{\phi},
$$

where $H_{m}$ is a typical vertical scale of variation of the profile function $g(z)$. This function is taken to be 1 in the upper layer and zero below so that $H_{m}$ is typically the depth of the upper layer.
In the present configuration, a linear friction parameterization is adopted:

$$
\left(\mathcal{F}^{u}, \mathcal{F}^{v}\right)=-\nu(u, v) .
$$

The choice for Rayleigh friction, instead of a harmonic formulation, relaxes the need to resolve narrow frictional boundary layers. We have to face that in no way the dynamics of the western boundary currents can be realistically represented in a coarse-resolution model context as applied here. Nonetheless, linear friction gives rise to Stommel boundary layers, whose thicknesses vary with latitude along with $\beta$. With the grid spacing in the zonal direction varying with $\cos \phi$ as well, the boundary layer is worst resolved in equatorial regions. In order to minimize friction while still resolving the spatial scales required by the Stommel boundary layer thickness, the friction parameter $\nu$ is taken to depend on latitude according to

$$
\nu(\phi)=\nu_{0} \cos ^{2} \phi .
$$

Density $\rho$ is related to temperature and salinity via an equation of state that is linear in salinity and thirdorder in temperature. It is based on the polynomial expression by Winton and Sarachik (1993):

$$
\rho(T, S)=\rho_{0}\left(1+a_{1} S-b_{1} T-b_{2} T^{2}+b_{3} T^{3}\right) .
$$

Thermohaline forcing is accomplished by restoring temperature and salinity to observed fields of annual mean temperature $T_{L}$ and salinity $S_{L}$ as compiled by Levitus and Boyer (1994) and Levitus et al. (1994), hereinafter 
jointly referred to as L94. These fields are interpolated onto the model grid and are smoothed using Laplace filtering. This defines the source terms $Q_{T}$ and $Q_{S}$ in Eqs. (1e) and (1f), which will be specified below. Using these source terms, the appropriate boundary conditions for temperature, salinity, and wind stress at the oceanatmosphere boundary are no-flux conditions.

Note that the model formulated here does not guarantee stably stratified solutions. As in all other largescale ocean models the effect of convection, which occurs when the stratification is statically unstable, must be explicitly parameterized. Such a parameterization is usually referred to as "convective adjustment." Several parameterizations can be used for this model, such as the locally implicit mixing parameterization and the global adjustment procedure (Weijer and Dijkstra 2001). However, their use is often awkward and time consuming, and, since a completely stable stratification is not crucial for the existence of the oscillatory modes, no convective adjustment is applied in the results below.

The implicit formulation of the model enables the direct computation of steady states without the need for laborious time integrations. Instead, steady states are followed through parameter space using so-called pseudoarclength continuation (Keller 1977). The procedure starts off with the zero solution and the forcing set to zero. The steady state is followed when increasing the forcing amplitudes with small steps until the full-forcing case is reached. The sensitivity of a solution to parameterizations can easily be studied by continuation in the direction of the relevant parameters. Furthermore, bifurcations can be detected, which are indicative of qualitative changes to the steady solutions. Examples are saddle-node bifurcations (also known as limit points) and Hopf bifurcations, where periodic solutions arise.

Last, each solution can be checked for its (linear) stability by solving a generalized eigenvalue problem of the form

$$
\mathcal{A} \mathbf{u}=\varsigma \mathcal{B} \mathbf{u} .
$$

This problem arises by perturbing the steady state with infinitesimally small perturbations and by considering the evolution of these perturbations. Two methods are currently available to solve Eq. (6): the Jacobi-Davidson QZ method (Sleijpen and Van der Vorst 1996) and the Simultaneous Iteration Method (e.g., Dijkstra 2000); the latter is used here. The procedure returns a specified number (say, 10) of least stable eigenmodes, with eigenvectors $\mathbf{u}$ (or $\mathbf{u}_{r}+i \mathbf{u}_{i}$ when a complex conjugate pair is found) and their eigenvalues $\boldsymbol{s}=\boldsymbol{s}_{r}+i \boldsymbol{s}_{i}$. Note that while the amplitudes of the resulting eigenvectors are-in principal-undetermined, the relative amplitudes of the dynamical fields $(T, S, u, v, w$, and $p$ ) within each eigenvector are internally consistent.

The set in Eq. (1) and the associated eigenvalue problem in Eq. (6) are discretized in space using a controlvolume discretization method on a staggered (Arakawa C) grid, which is second-order accurate.
TABLE 1. Values of parameters used in the calculations.

\begin{tabular}{|c|c|c|c|c|c|c|}
\hline$=$ & $1.46 \times 10^{-4}$ & $\left(s^{-1}\right)$ & $r_{0}$ & $=$ & $6.37 \times 10^{6}$ & (m) \\
\hline$=$ & $1.0 \times 10^{3}$ & $\left(\mathrm{~kg} \mathrm{~m}^{-3}\right)$ & $H_{m}$ & $=$ & 60 & $(\mathrm{~m})$ \\
\hline$=$ & $4.0 \times 10^{3}$ & $\left(\mathrm{~m}^{2} \mathrm{~s}^{-1}\right)$ & $\kappa_{v}$ & $=$ & $2.0 \times 10^{-4}$ & $\left(\mathrm{~m}^{2} \mathrm{~s}^{-1}\right)$ \\
\hline$=$ & $1.46 \times 10^{-5}$ & $\left(s^{-1}\right)$ & $H$ & $=$ & $5.0 \times 10^{3}$ & (m) \\
\hline$=$ & $4.2 \times 10^{3}$ & $\left(\mathrm{~J} \mathrm{~kg}^{-1} \mathrm{~K}^{-1}\right)$ & $g$ & $=$ & 9.8 & $\left(\mathrm{~m} \mathrm{~s}^{-2}\right)$ \\
\hline$=$ & $7.6 \times 10^{-4}$ & $\left(\mathrm{psu}^{-1}\right)$ & $b_{1}$ & $=$ & $5.6 \times 10^{-5}$ & $\left(\mathrm{~K}^{-1}\right)$ \\
\hline$=$ & $6.3 \times 10^{-6}$ & $\left(\mathrm{~K}^{-2}\right)$ & $b_{3}$ & $=$ & $3.7 \times 10^{-8}$ & $\left(\mathrm{~K}^{-3}\right)$ \\
\hline$=$ & $3.6 \times 10^{-1}$ & $\left(\mathrm{~N} \mathrm{~m}^{-2}\right)$ & $\tau_{s}$ & $=$ & 74 & (days) \\
\hline
\end{tabular}

\section{Results}

\section{a. The basic steady solution}

The model domain covers the World Ocean between $75^{\circ} \mathrm{S}$ and $75^{\circ} \mathrm{N}$. The model grid consists of $N \times M \times$ $L=72 \times 30 \times 14$ boxes, yielding a horizontal resolution of $5^{\circ} \times 5^{\circ}$. With six variables, this yields a dynamical system of over 180000 degrees of freedom. The grid is stretched in the vertical with layer depths ranging from $60 \mathrm{~m}$ at the surface to $736 \mathrm{~m}$ at the bottom. The bathymetry is derived from the ETOPO-10 dataset, which is interpolated onto the model grid and smoothed. Standard values of the parameters are listed in Table 1. Although the mixing of heat and salt is modeled in a crude way by just assuming constant horizontal and vertical exchange coefficients, the values listed in Table 1 are typical for low-resolution ocean models. With these parameters, the inertial terms in Eqs. (1a) and (1b) are small and can be neglected.

In the surface layer, temperature and salinity are restored to the L94 dataset with a time constant of 74 days. However, it turns out that, in that case, no steady state exists for the parameter values at this resolution. Unresolved processes (mainly in the Southern Ocean) give rise to grid-scale fluctuations that defy the presence of a steady solution. Indeed, time integrations show that pronounced variability is present. To obtain a steady state for the parameter values listed in the table, we retain a very weak restoring condition on temperature in the interior as well.

Hence, the formulation of the restoring terms is divided into a surface component that applies to the surface layer only and, for temperature, an internal term that applies to the subsurface ocean. The source terms in Eqs. (1e) and (1f) then become

$$
\begin{aligned}
& Q_{T}=\left(T_{L}-T\right)\left[\frac{g(z)}{\tau_{s}}+\frac{1-g(z)}{\tau_{d}(z)}\right] \\
& Q_{S}=\left(S_{L}-S\right) \frac{g(z)}{\tau_{s}} .
\end{aligned}
$$

Here, $\tau_{s}$ is the timescale for surface relaxation and is taken equal for heat and salt, while $\tau_{d}$ controls the internal relaxation. This timescale is taken inversely proportional to the grid spacing at depth and ranges from $11.5 \mathrm{yr}$ for the subsurface layer to $108 \mathrm{yr}$ for the deepest layer. 
a)

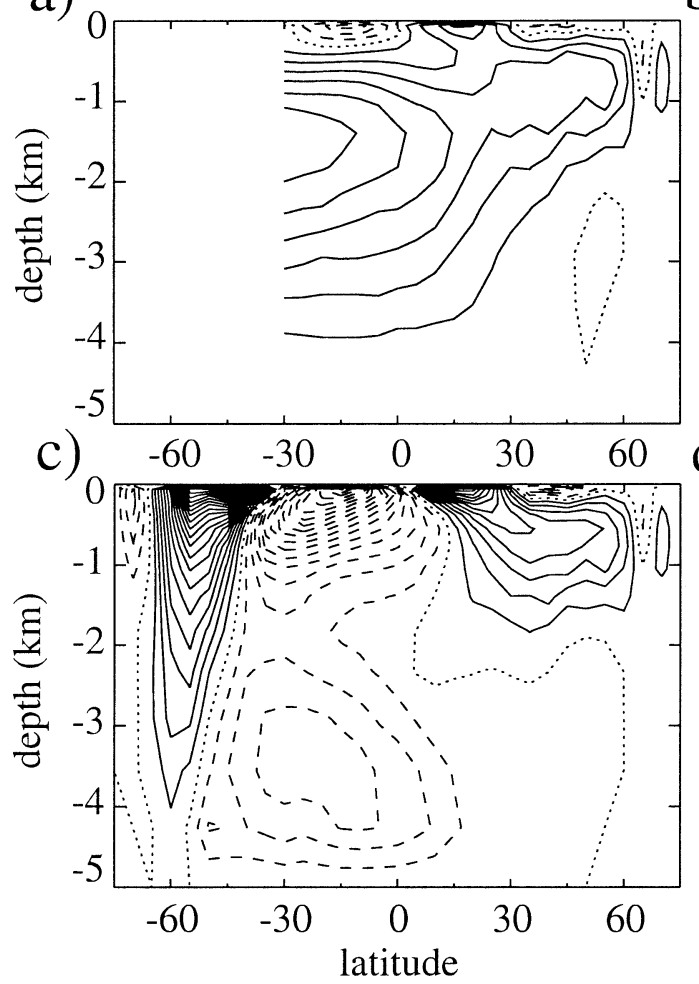

b)

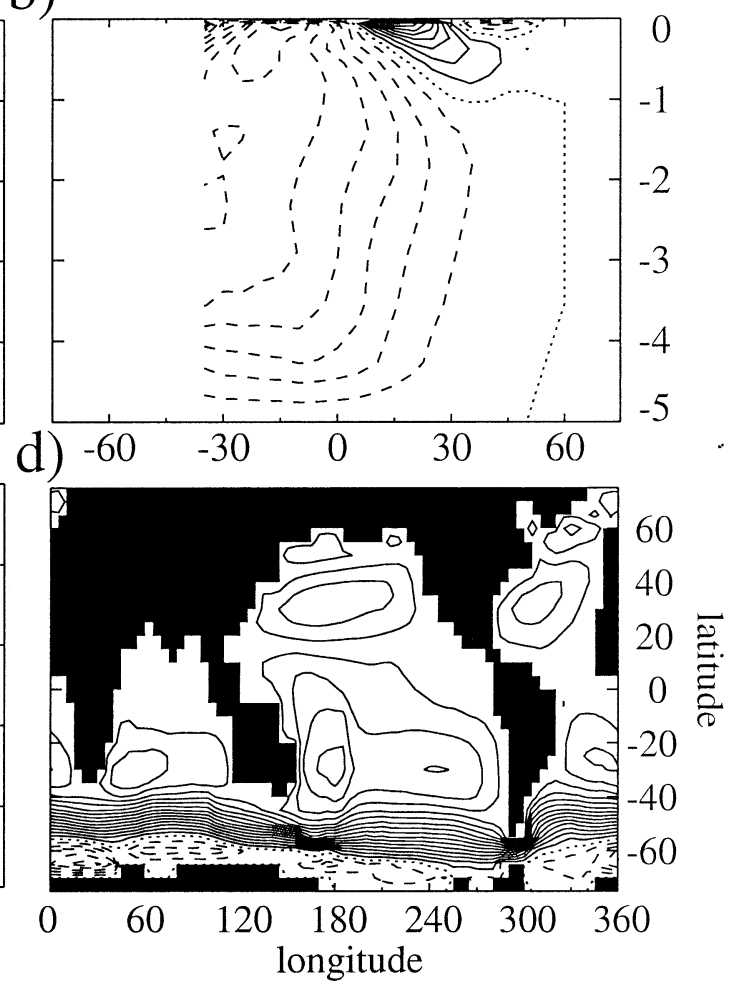

FIG. 1. The (a) Atlantic, (b) Pacific, and (c) global overturning streamfunctions and (d) global barotropic streamfunction for the steady solution. Positive, zero, and negative contours are solid, dotted, and dashed, respectively. Contour interval is $2 \mathrm{~Sv}$ in (a)-(c) and $5 \mathrm{~Sv}$ in (d).

The steady solution (Fig. 1) shows a decent global circulation. It features a strong downwelling cell in the Atlantic (Fig. 1a), whereas in the Pacific large-scale upwelling takes place (Fig. 1b). There is $13.5 \mathrm{~Sv}(\mathrm{~Sv}$ $\equiv 10^{6} \mathrm{~m}^{3} \mathrm{~s}^{-1}$ ) outflow of water deeper than $1500 \mathrm{~m}$ from the Atlantic into the rest of the World Ocean, whereas there is 12 and $5.5 \mathrm{~Sv}$ of upwelling across this level in the Pacific and Indian Oceans, respectively. The excess of $4 \mathrm{~Sv}$ deep-water upwelling in these basins must have its origin south of $30^{\circ} \mathrm{S}$. If interpreted as Antarctic Bottom Water (AABW), this amount agrees well with observed estimates (Broecker et al. 1998). The

TABLE 2. The timescales of decay $\left(\tau_{\text {decay }}=1 / \varsigma_{r}\right)$ and oscillation ( $\left.\tau_{\text {oscillation }}=2 \pi / s_{i}\right)$ of the least stable eigenvectors of the basic solution. Eigenmodes 1, 3, and 7 are complex, indicating oscillatory instabilities, whereas modes 2, 4, 5, and 6 are real. Symbols in fourth column correspond to those used in Figs. 8 and 9.

\begin{tabular}{cccc}
\hline \hline No. & $\tau_{\text {decay }}(\mathrm{yr})$ & $\tau_{\text {oscillation }}(\mathrm{yr})$ & Symbol \\
\hline 1 & 380 & 3827 & $\square$ \\
2 & 329 & & $\mathbf{0}$ \\
3 & 202 & 2372 & $\bullet$ \\
4 & 179 & & $\mathbf{\Delta}$ \\
5 & 138 & & $\diamond$ \\
6 & 135 & 2674 & $\diamond$ \\
7 & 105 & & \\
\hline
\end{tabular}

main deficit of the present steady circulation is the absence of bottom water in the Atlantic basin.

The barotropic streamfunction (Fig. 1d) represents the basic structures of the main subtropical and subpolar gyres. Most gyres display a transport exceeding $10 \mathrm{~Sv}$, while the South Pacific gyre transports over $20 \mathrm{~Sv}$. The most prominent feature, however, is the Antarctic Circumpolar Current. Its strength is $60 \mathrm{~Sv}$, which is about one-half as strong as suggested by observations (Nowlin and Klinck 1986).

\section{b. Linear stability analysis and mode characteristics}

For solving the eigenvalue problem in Eq. (6) associated with the linear stability of the steady state of the previous section, the relaxation terms at the surface and in the interior are set to zero and no-flux conditions are maintained at the surface. The resulting modes are hence not affected by the restoring terms of the steady state.

In Table 2, the eigenvalues of the seven least stable modes are presented as the associated timescales of decay and oscillation. Two types of eigenmodes are present: modes 2, 4, 5, and 6 are real, whereas 1, 3, and 7 are complex conjugate pairs. The latter are indicative of internal oscillatory modes of variability. 
Atlantic
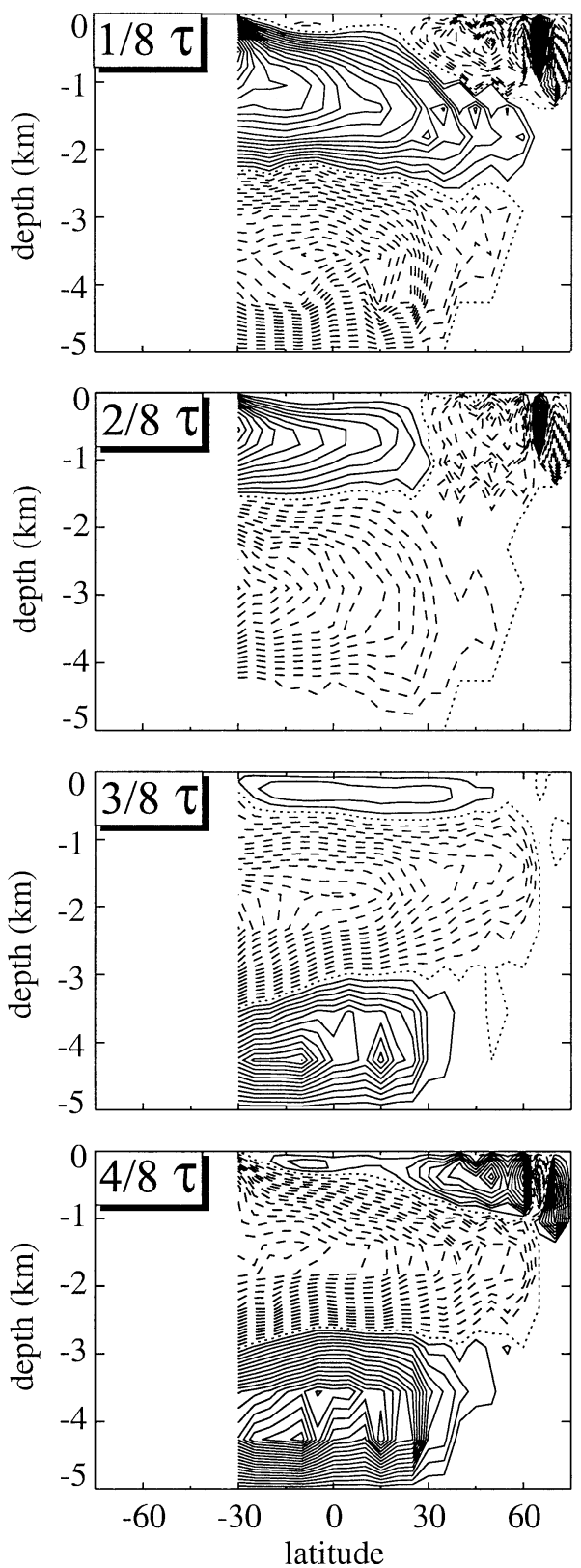

Pacific
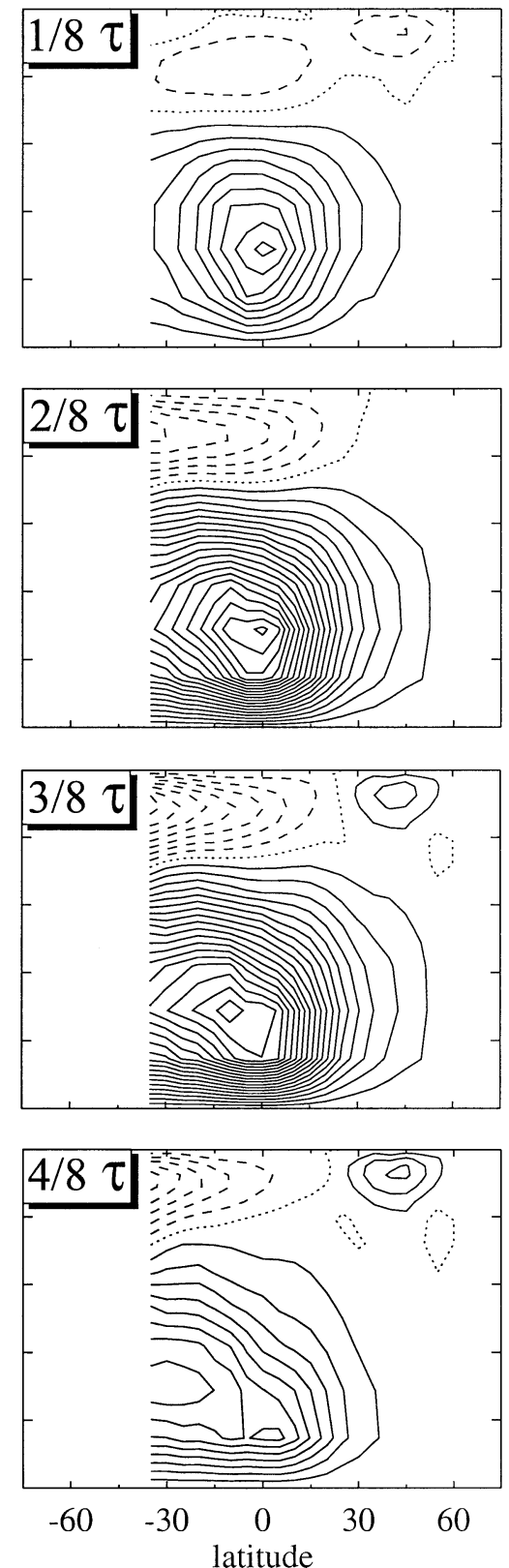

FIG. 2. Anomalous overturning streamfunction in the (left) Atlantic and (right) Pacific for the oscillatory mode 1. Shown are four snapshots during the first half of the oscillation, 1/8 of an oscillation period apart. The second half is identical to the first, albeit with signs reversed. Note that the amplitudes of the eigenvectors are undetermined; although the contour interval is the same for all panels, absolute values of the contours are not relevant.

The fact that all eigenvalues have negative real parts shows that the steady solution is linearly stable. But when the steady state is disturbed and the time evolution of the perturbation is determined, these eigenmodes will take longest to decay. Consequently, in the transient response of the circulation to randomly applied disturbances, for instance induced by stochastic components in the forcing, these modes are expected to dominate in the long term response (Griffies and Tziperman 1995).

Note that the patterns of the eigenmodes are fully consistent with the governing equations-they satisfy the equations linearized around the steady state-and boundary conditions. In this way, they differ completely from empirical orthogonal functions (EOF) or other sta- 
tistically determined modes, which lack the property above [a probable exception being the empirical normal modes, as developed by Brunet (1994)]. The eigenmodes are therefore much more appropriate to determine physical mechanisms of the propagation and growth of specific perturbations. This motivates to look at characteristics of the patterns in the next subsections.

\section{1) Meridional overturning}

Oscillatory mode 1 has a period $\tau$ of $3827 \mathrm{yr}$ at standard values of the parameters. It displays interesting anomalies in the overturning streamfunction (Fig. 2). At phase $t=(1 / 8) \tau$, a positive anomaly overlies a negative anomaly in the Atlantic. The boundary between these cells can be seen to migrate upward in time, until one half of a period later a negative anomaly overlies a positive one. The Pacific overturning anomaly also features a layered pattern, with a negative cell overlying a positive one during the first half of the oscillation. Contrary to the Atlantic, this pattern does not migrate upward in time; it just amplifies $[t=(2 / 8) \tau$ and $(3 / 8) \tau]$, attenuates $[t=(4 / 8) \tau]$, and reverses sign in the second half of the oscillation.

Oscillatory mode 3, which has a period $\tau$ of $2372 \mathrm{yr}$, shows overturning anomalies that are similarly layered. But interestingly, in mode 3 the roles of the Atlantic and the Pacific seem to be reversed (Fig. 3); now the layered overturning structure in the Pacific seems to migrate upward, whereas the anomalies in the Atlantic display a standing pattern.

\section{2) Propagation of AnOMAlies}

A characteristic of loop oscillations is the propagation of thermohaline anomalies along the overturning circulation. The propagation of salinity anomalies displays the same pattern as the dispersion of their thermal counterparts. In fact, thermal and saline anomalies are of the same sign and largely, but not completely, density compensating throughout the oscillations. Hence, only the thermal anomalies are shown. For modes 1 and 3 it is shown here that anomalies indeed follow the path of the global overturning circulation. The journey of a thermal anomaly can best be followed through the zero-phase line $\left\{T^{\prime}=0 \mid T_{t}^{\prime}>0\right\}$ ("the anomaly") that separates negative anomalies (denoted blue) from positive anomalies (red).

For mode 1, the phase line starts off in the deep North Atlantic $[t=(1 / 16) \tau$, Fig. 4] and is advected southward by the deep branch of the Atlantic overturning. It reaches the Southern Ocean $[t=(2 / 16) \tau]$ and spreads out into the deep Indian Ocean $[t=(3 / 16) \tau]$. Although the propagation west of the date line is fairly rapid, it takes a while before the anomaly enters the deep Pacific. At this depth, the gap between Tasmania and Antarctica is as wide as Drake Passage in this model $\left(10^{\circ}\right)$, and it is probable that this submarine topography inhibits the interaction between the Indian and Pacific sectors of the
Southern Ocean. In fact, although Drake Passage is open at this model level, no anomalies pass through this conduit. It takes until $t=(7 / 16) \tau$ before the anomaly sets out for the Pacific and at $t=(9 / 16) \tau$ it finally reaches its northern rim. Here, it takes some time before it surfaces $[t=(12 / 16) \tau$ in Fig. 5]. Once picked up by the upper branch of the overturning circulation, the anomaly spreads out rapidly, and reaches the North Atlantic already at $t=(14 / 16) \tau$.

Similarly for mode 3, the anomaly (Figs. 6 and 7) starts off in the deep North Atlantic $[t=(2 / 16) \tau]$ and, when it reaches the Southern Ocean, it propagates eastward $[t=(6 / 16) \tau]$ and fills the deep Indian $[t=(9 /$ $16-11 / 16) \tau]$ and Pacific Oceans $[t=(14 / 16-19 / 16) \tau]$. But rather than a single anomaly traveling through the deep ocean, two anomalies are present in the abyss at the same time. So when a positive anomaly sets off in the deep North Atlantic $[t=(2 / 16) \tau$, Fig. 6], another anomaly is still working its way north in the deep Pacific. With two anomalies running after each other through the abyssal ocean, anomalies are generated in the shallow North Pacific twice as often as in mode 1. If one considers mode 1 as an " $n=1$ " mode, where $n$ is referring to the number of zeroes of an eigenfunction in classical stability problems, such as the Eady problem (Pedlosky 1987), then mode 3 can be considered as an " $n=2$ " mode. Indeed, the ratio of 0.6 between the periods of modes 3 and 1 comes close to the 0.5 that would be expected for such a mode.

From the propagation characteristics it is clear that the oscillatory period is determined by the time it takes for thermal (and saline) anomalies to travel the deep branch of the overturning circulation. This can be regarded as the slow phase of the oscillation. Indeed, for mode 1 it takes exactly one-half (one-quarter for mode 3 ) of an oscillatory period for the zero phase-line to be carried from the North Atlantic into the deep North Pacific. The passage between Tasmania and Antarctica seems to cause the most delay and is the slowest part of this journey. The propagation of the anomalies at shallow levels, on the contrary, is much faster (close to "instantaneous," when compared with the oscillation period). This is the rapid phase of the oscillation, and it hardly influences the timescale.

\section{3) LINKING THE DEEP AND SHALLOW BRANCHES}

From the patterns, one observes that the deep and shallow branches of the global journey of the anomalies are linked in the North Pacific and North Atlantic Oceans, but the details of this link need further analysis. What generates the anomalies in the deep North Atlantic, and what causes anomalies to emerge in the shallow North Pacific? What determines the time lag between the arrival of an anomaly in the shallow North Atlantic and the generation of an anomaly at depth (vice versa for the North Pacific)?

It seems that several advective mechanisms are im- 
Atlantic
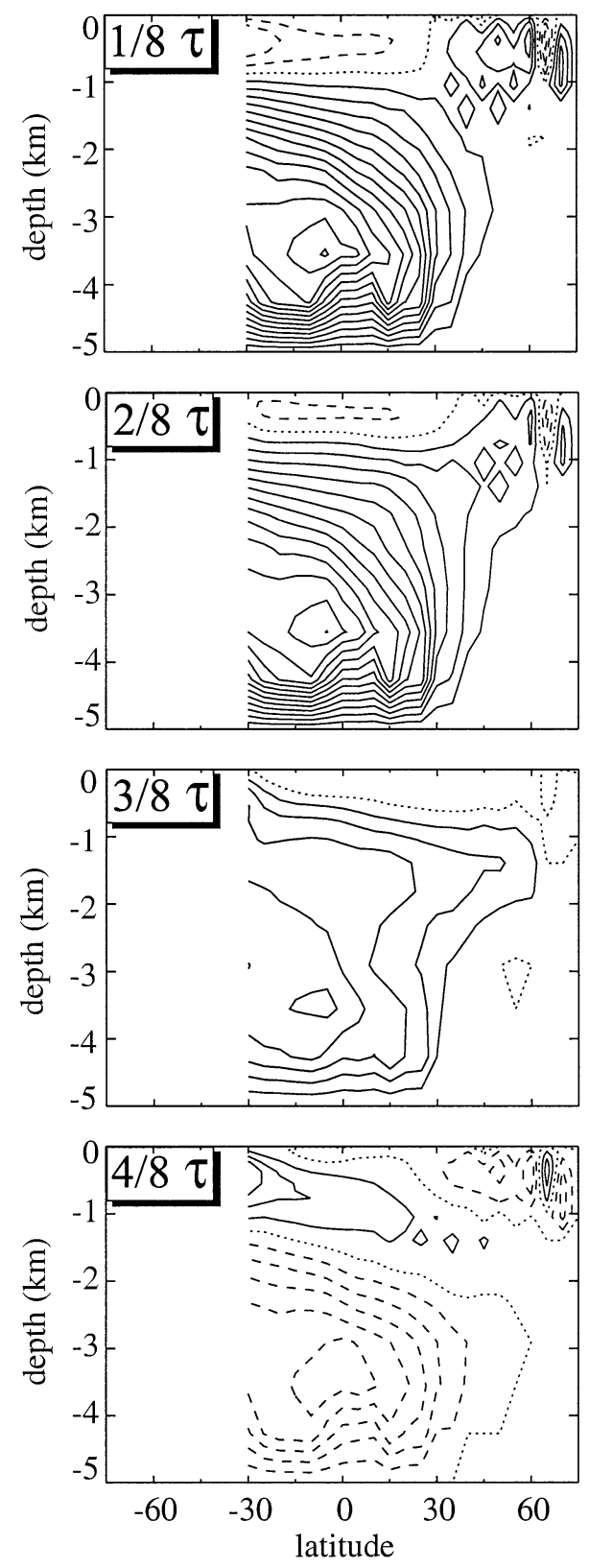

Pacific
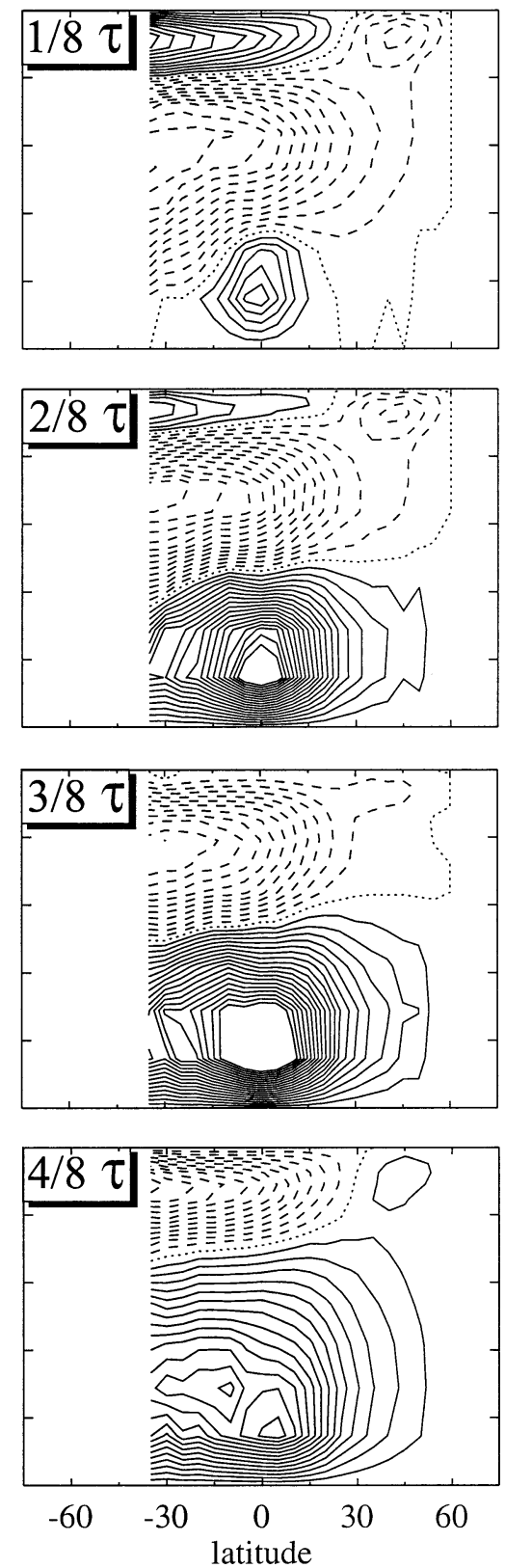

FIG. 3. As in Fig. 2 but now for oscillatory mode 3.

portant for the propagation of the anomalies. First there is northward advection of the warm anomaly, coming in from the Pacific, that warms the shallow layers of the North Atlantic. Downwelling of this anomaly by the steady-state overturning warms the deep layers, which are at a temperature minimum at that point. The time lag between arrival of the positive anomaly at shallow levels and the generation of a positive anomaly at depth is set by this downwelling. In addition, another advective mechanism is present that assists in warming the deep layers and cooling the shallow anomaly. At the time of arrival of the shallow positive temperature anomaly, an overturning anomaly is present that features a negative anomaly overlying a positive one [cf. the pattern depicted in Fig. 2; $t=(3 / 8) \tau]$; the upwelling at shallow levels advects cold water upward, and cools the anomaly, while at depth the downwelling warms the deep layers. This mechanism hence amplifies the deep anomaly, counteracting the ubiquitous attenuation by diffusion. 

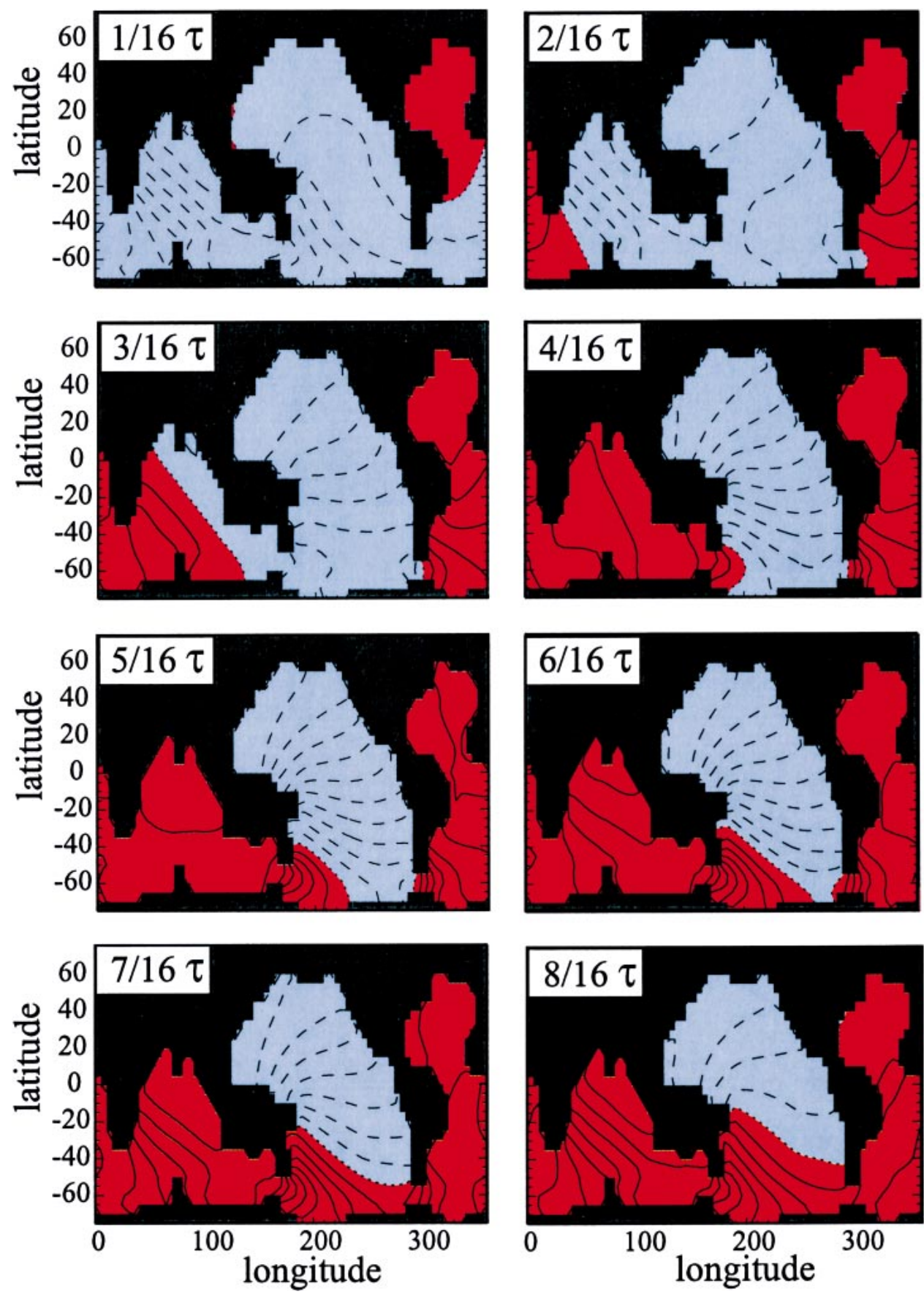

FIG. 4. Mode-1 propagation of temperature anomalies at 3000-m depth. Positive (negative) anomalies are denoted by solid (dashed) contours and red (blue) colors. Note again that the amplitudes are undetermined, but understood to be small. Contour levels are the same for all panels.

Also in the North Pacific, a phase differences exist between anomalies at depth and at shallow levels. A positive anomaly emerges at shallow levels when the deep anomaly has reached its maximum. Again, basicstate upwelling plays a role in warming the upper layers. But also in the North Pacific does the anomalous over- turning amplify the positive anomaly at shallow levels; its layered structure, at this time with a negative cell overlying a positive one [Fig. $2 ; t=(1 / 8) \tau$ ], tends to warm the upper $1500 \mathrm{~m}$. At deeper levels, where the background temperature gradient is small, its contribution is negligible. 

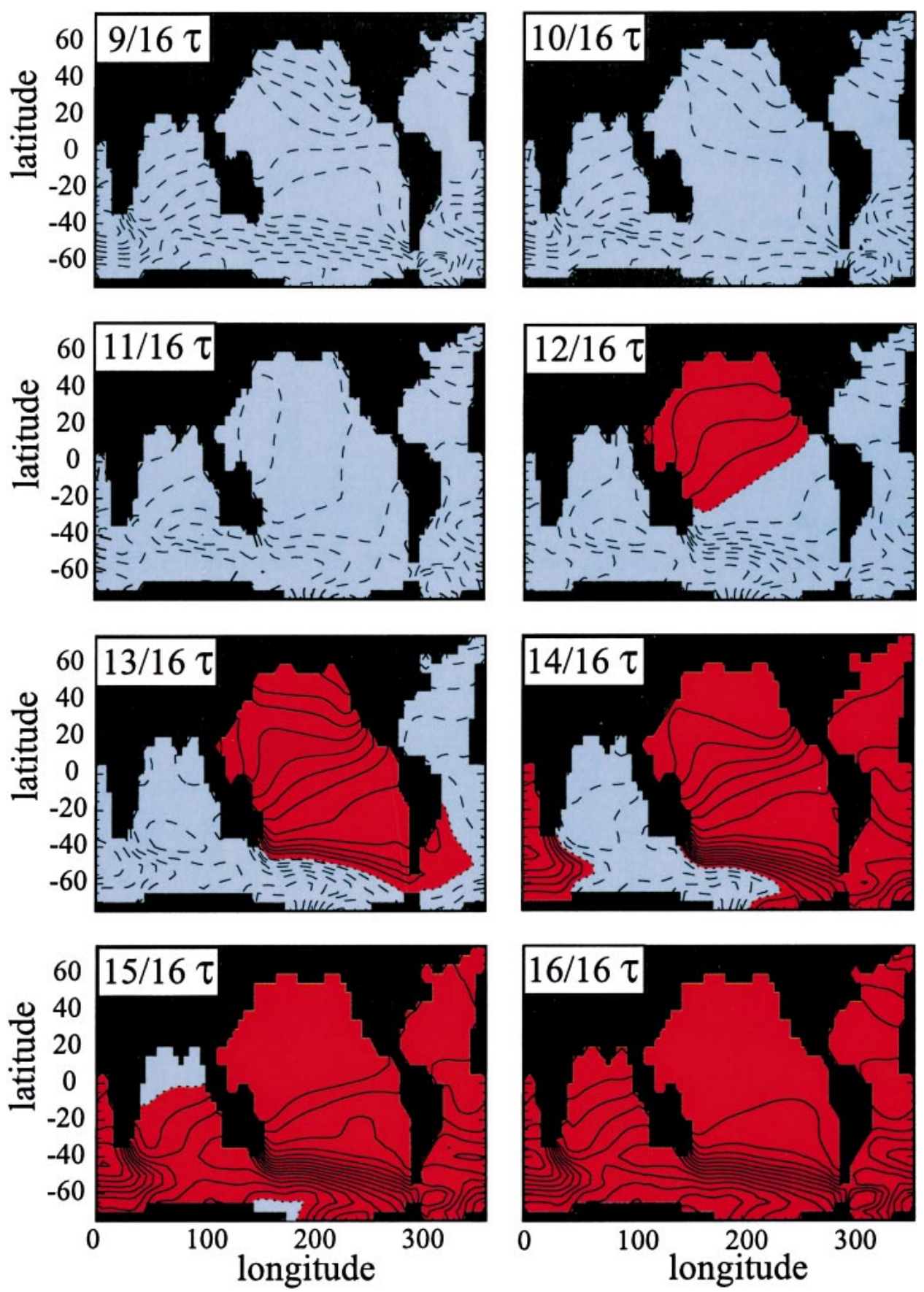

FIG. 5. Mode-1 propagation of temperature anomalies at 500-m depth. Contouring and coloring conventions are as in Fig. 4. Note that the second half of the oscillation is displayed, following on the sequence shown in Fig. 4.

\section{c. Physical mechanism}

Ideally, the mechanism of an oscillation is described in terms of conversions between potential and kinetic energy anomalies (e.g., Te Raa and Dijkstra 2002). However, the modes do not become neutral and, hence, the methodology to arrive at a consistent description of the oscillatory mechanism based on energy conversions cannot be used. In this section, another method is applied to infer the importance of certain terms for the dynamics of the oscillation: the strength of the several feedbacks is controlled by changing relevant parameters in the eigenvalue calculations. 

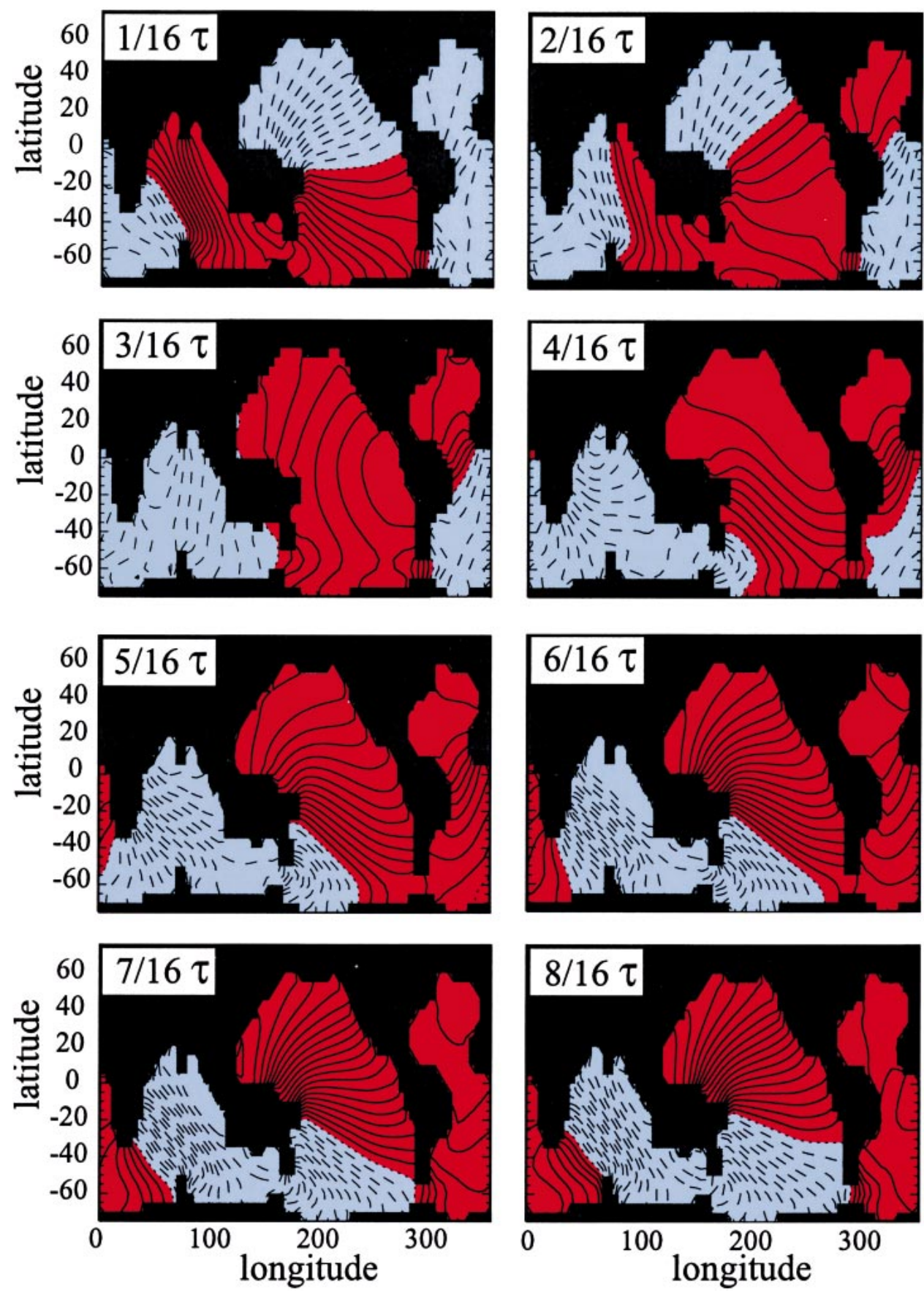

FIG. 6. Mode-3 propagation of temperature anomalies at 3000-m depth. Contouring and coloring conventions are as in Fig. 4.

Thermal (and saline) anomalies satisfy the following equation:

$$
\frac{\partial T^{\prime}}{\partial t}=\mathcal{F}_{1}+\mathcal{F}_{2}+\kappa_{h} \nabla_{h}^{2} T^{\prime}+\kappa_{v} \frac{\partial^{2} T^{\prime}}{\partial z^{2}},
$$

with

$$
\mathcal{F}_{1}=-\mu_{1} \overline{\mathbf{u}} \cdot \boldsymbol{\nabla} T^{\prime} \text { and } \quad \mathcal{F}_{2}=-\mu_{2} \mathbf{u}^{\prime} \cdot \nabla \bar{T},
$$

where the steady solution is denoted by an overbar and the anomalies by a prime, and where $\mu_{1}=\mu_{2}=1$. For convenience, the interaction between the background flow and thermal (and saline) anomalies will be denoted as $\mathcal{F}_{1}$, whereas the interaction between the flow anomalies and the background thermal (and saline) stratification as $\mathcal{F}_{2}$.

To determine the importance for each of the terms on 

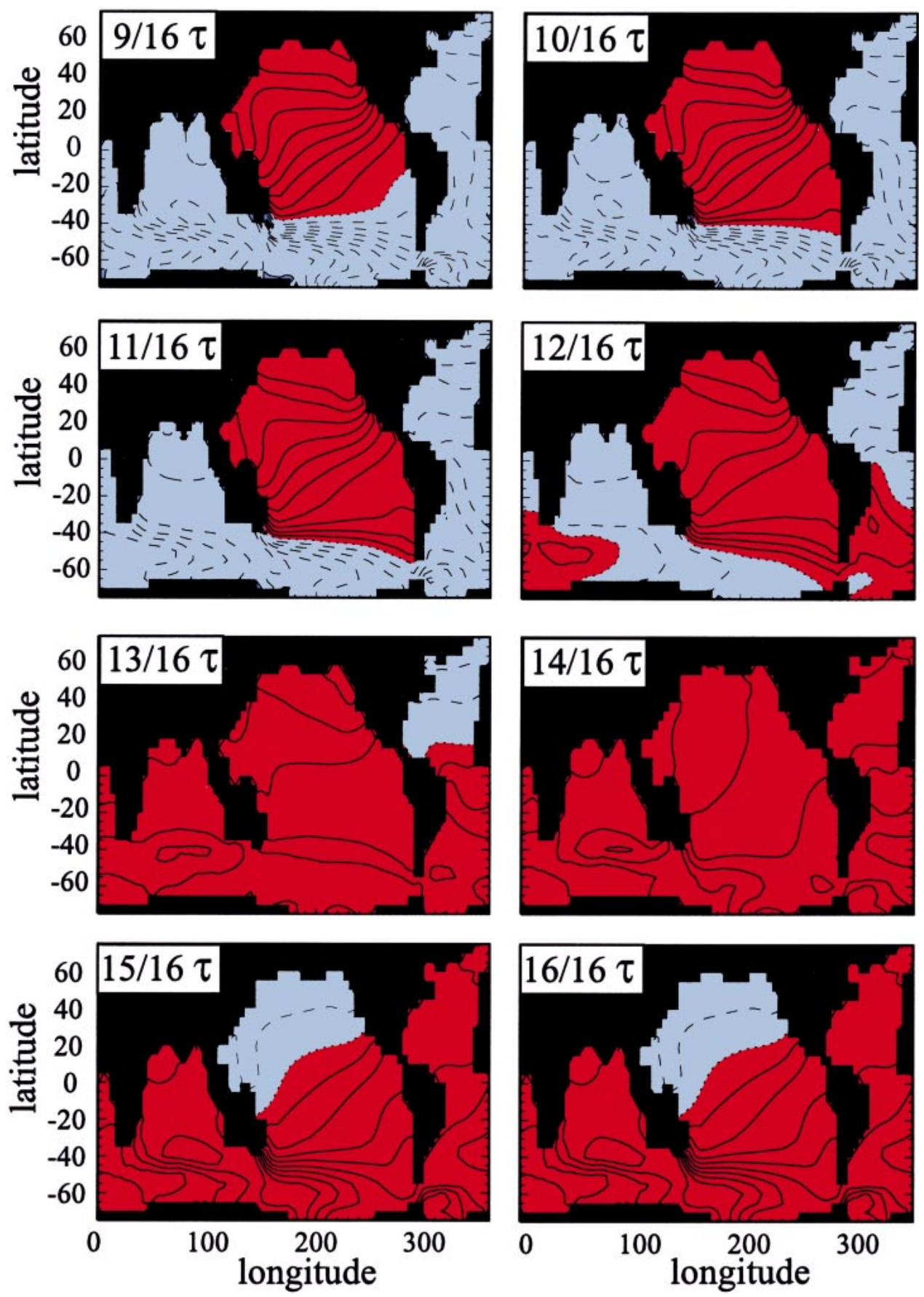

FIG. 7. Mode-3 propagation of temperature anomalies at 500-m depth. Contouring and coloring conventions are as in Fig. 4.

the growth rate and period of the oscillatory modes, parameters are varied in the linear stability problem while the steady state is fixed. In particular, the parameters $\mu_{1}$ and $\mu_{2}$ are introduced to control the strength of the advective feedbacks. A strong response of either $\boldsymbol{s}_{r}$ or $\boldsymbol{s}_{i}$ to changing a parameter may identify the corresponding feedback as being essential for either decay or oscillation of the eigenmode. The advantage is that a change in the eigenvalues or -vectors can be due only to (controlled) changes in the dynamics, and not to changes in the basic state. This allows for a more accurate identification of the relevant mechanisms. Besides, we do not need to calculate a new basic state for each new value of the parameters, making the procedure computationally very efficient.

In the appendix, it is shown how the growth rates and 


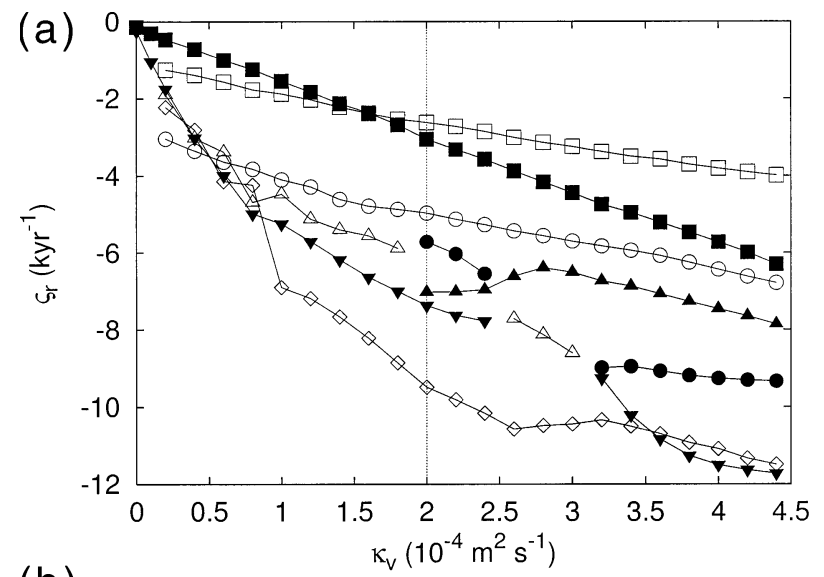

(b)
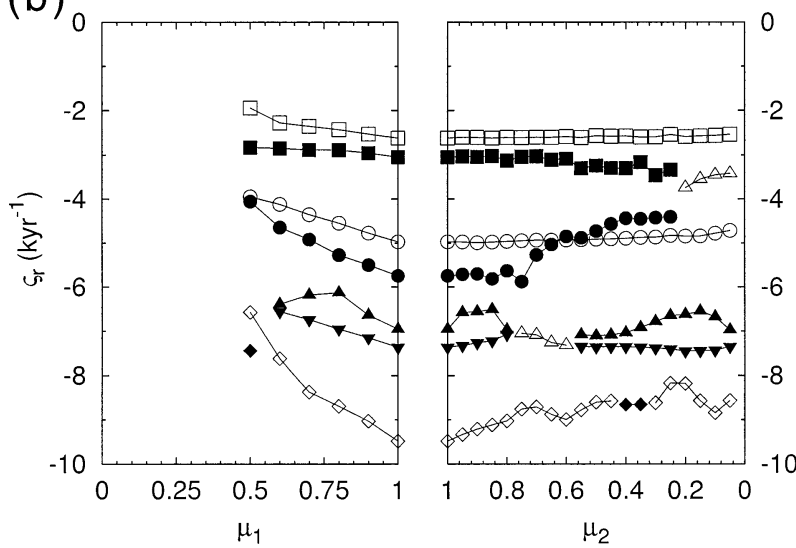

FIG. 8. Real parts of the eigenvalues of the least stable eigenmodes as function of (a) the vertical diffusivity $\kappa_{v}$ and (b) the advective feedback parameters $\mu_{1}$ and $\mu_{2}$. Note that $\varsigma_{r}$ is inversely proportional to growth (decay) rate. For $\kappa_{v}<0.2 \mathrm{~m}^{2} \mathrm{~s}^{-1}$, only the eigenvalues of the two dominant real modes could be determined with sufficient accuracy, whereas no accurate eigenvalues could be computed for $\mu_{1}$ $<0.5$. Open (solid) symbols represent complex (real) modes. Vertical line in (a) denotes value of vertical diffusivity of the basic steady solution; modes on this line correspond to modes listed in Table 2.

oscillation periods can be expressed in terms of volume integrated quantities involving the interactions between the spatial patterns of the eigenmode and the background steady state. For some terms the parameter dependence is explicit, while for others it is implicit; a change in an implicit parameter may work through via the direct terms when it affects the eigenfunctions. Equation (A5a) shows that $\boldsymbol{s}_{r}$ may depend directly on $\mathcal{F}_{2}$ and on the diffusion terms, but not on $\mathcal{F}_{1}$. Advection of anomalies by the background flow can only modify $\varsigma_{r}$ indirectly, when it changes the spatial structure of the eigenmodes and hence affects the diffusive feedbacks or $\mathcal{F}_{2}$. Similarly, $s_{i}$ directly depends on both advective feedbacks only [Eq. (A5b)]; diffusion can only change the oscillation timescale indirectly, when it changes the spatial patterns and thereby the strength of the advective feedbacks. Oscillatory motion therefore only exists in the presence of advective feedbacks: diffusion may
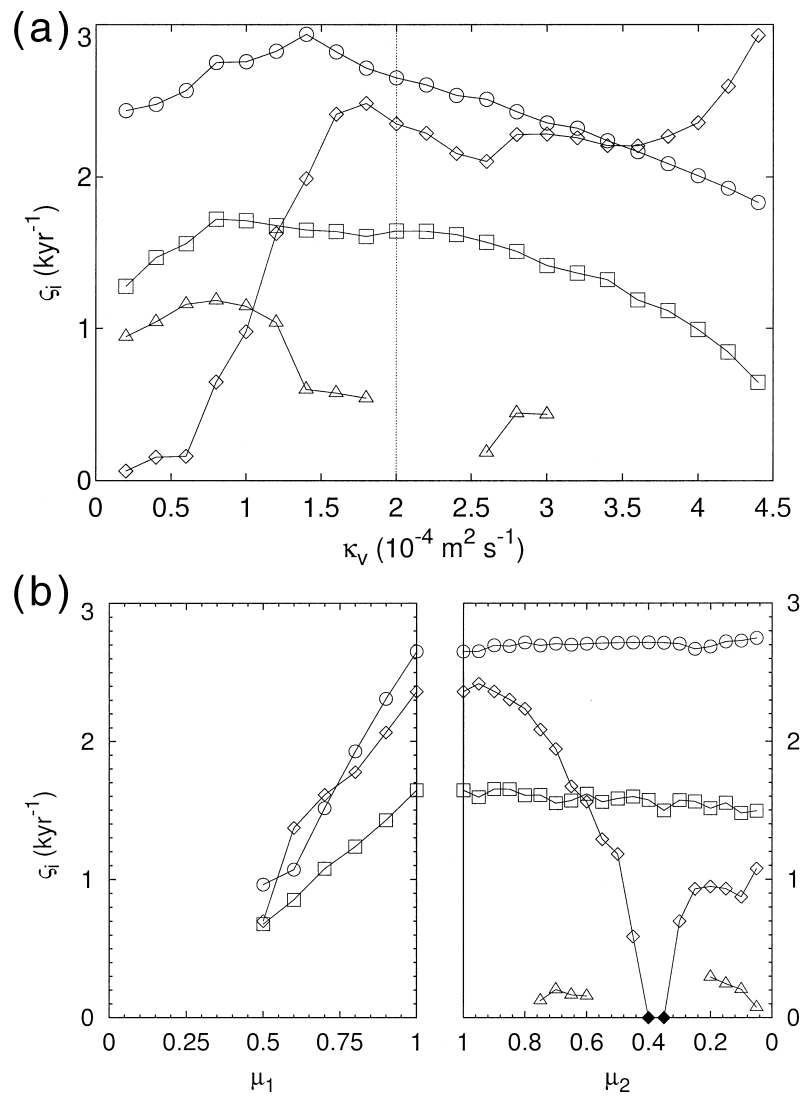

FIG. 9. As in Fig. 8 but now for the imaginary parts of the eigenvalues. Note that $\boldsymbol{s}_{i}$ is inversely proportional to the oscillation period.

modify the timescale; however, it can never be responsible for oscillatory motion in this system.

In Fig. 8, the growth rates of the least stable modes are shown as a function of $\kappa_{v}, \mu_{1}$, and $\mu_{2}$. The almost linear relationship between $\boldsymbol{S}_{r}$ and $\kappa_{v}$ shows that vertical diffusion strongly controls the growth rate of all modes. Especially some of the real modes closely approach $\boldsymbol{s}_{r}$ $=0$ for $\kappa_{v} \rightarrow 0$. The oscillatory modes seem to remain stable for $\kappa_{v}=0$, although we could not determine these modes with sufficient accuracy here. On the other hand, there is only weak response to a change in the advective feedback parameters $\mu_{1}$ and $\mu_{2}$ (Fig. 8b) as well as to the horizontal mixing coefficient $\kappa_{h}$ (not shown).

Vertical diffusivity influences the oscillation periods of modes 1 and 3 to some extent, albeit with no clear systematic trend (Fig. 9a). For higher values of $\kappa_{v}$, modes 1 and 3 display a tendency of increasing $\boldsymbol{s}_{i}$ (shorter periods) for decreasing $\kappa_{v}$, whereas for lower values of $\kappa_{v}$ this tendency is reversed. The response of mode 7 (open diamonds) is very strong, showing a spectacular drop in $\varsigma_{i}$ when $\kappa_{v}$ is reduced. The period increases from $2.5 \mathrm{kyr}$ for $\kappa_{v}=1.8 \times 10^{-4} \mathrm{~m}^{2} \mathrm{~s}^{-1}$ to almost $10 \mathrm{kyr}$ for small $\kappa_{v}$. According to Eq. (A5b), the response of the oscillation periods must be ascribed to changes in the advective feedbacks caused by modifications of the 
spatial patterns of the eigenmodes. The response of $\boldsymbol{s}_{i}$ to changes in $\kappa_{h}$ is very weak (not shown).

On the other hand, the periods of the oscillatory modes do show a particularly strong (and linear) response to a change in $\mu_{1}$ (Fig. 9b). This parameter controls the amplitude of advection by the background flow. This dependency indicates that the timescale of the oscillations is largely set by the advection timescale of the steady flow. Again, mode 7 is exceptional by also responding strongly to a change in $\mu_{2}$ as well. This most stable mode of the ones presented here shows an enormous decrease in $\boldsymbol{s}_{i}$, and becomes a real mode for a short segment of $\mu_{2}$. This indicates that for this mode interaction between the background stratification and the anomalous flow is important as well.

This analysis demonstrates that the growth rate of the eigenmodes is clearly controlled by vertical diffusion. Although Eq. (A5a) shows that horizontal diffusion and the second advective feedback have the potential to influence the stability as well, they appear to play only a minor role. The oscillatory periods of the two least stable oscillatory modes 1 and 3, on the other hand, are clearly determined by the first advective feedback, that is, the advective timescale of the global circulation. Advection of anomalies by the global circulation $\left(\mathcal{F}_{1}\right)$ is hence the crucial element of the oscillation, as also concluded from the propagation of anomalies. The feedback $\mathcal{F}_{2}$ is of no importance for setting the timescale, despite its theoretical potential to do so [Eq. (A5b)]. These results qualify the modes to be global loop or overturning oscillations.

\section{Discussion and conclusions}

As far as we are aware, this is the first time that the linear stability of a global ocean flow has been calculated explicitly by directly solving the generalized eigenproblem. It resulted in the explicit identification of multiple internal oscillatory modes. It has been shown that the basic oscillatory mechanism of the two least stable oscillatory modes is the advection of thermal and saline anomalies over the global overturning oscillation. The timescale is set by the propagation of anomalies from the North Atlantic to the North Pacific at depth. The least stable mode (mode 1) has a period of 3827 yr, which is the time it takes for an anomaly to complete a full cycle over the global circulation. In the second oscillatory mode (mode 3 ) two anomalies are traveling the global circulation at the same time. This almost halves the period to 2372 yr. Vertical mixing is the mechanism that controls the growth rate of these modes.

The overturning anomalies of modes 1 and 3 show an interesting layered structure in the Atlantic and Pacific Oceans. Since the temperature and salinity anomalies are nearly compensating, it may seem that the nonlinearity in the equation of state is crucial. Salinity anomalies would dominate density at depth, whereas at shallower levels thermal anomalies would be dominant.
So a given meridional temperature and salinity gradient of the same sign will generate a density gradient at depth that points in the same direction as the gradients of $T$ and $S$, whereas at shallower levels it opposes the gradients of $T$ and $S$. This reversal of the density gradient at middepth is reflected in the sign reversal of the anomalous overturning cells. However, it turns out the nonlinear equation of state is not essential for the existence of the oscillation. When the nonlinearity in the equation of state is reduced in the eigenvalue problem, there is no response in either the real or imaginary parts of the eigenvalues of modes 1 and 3. The nonlinear equation of state is therefore not responsible for the density anomalies that are required to generate anomalous flow nor are these anomalies caused by different boundary conditions on $T$ and $S$ anomalies (no-flux conditions were taken for both); they must be ascribed to the differences in the background distributions of $T$ and $S$. Interaction of flow anomalies with these different background distributions will generate the different thermal and saline anomalies that are required to generate density anomalies.

The fact that the steady state in the present model is linearly stable does not exclude the possibility that modes like the ones found here play a role in shaping climate variability. First of all, the present model configuration features relatively strong diffusion and relatively high friction in the equatorial regions. It is likely that the oscillatory modes are much less stable when more realistic values of the damping parameters are used. Indeed, Fig. 8a shows that the stability of the modes is strongly reduced when vertical diffusivity is decreased toward more realistic values. Second, damped oscillations may be excited by stochastic components in the forcing, like low-frequency variability of the atmospheric circulation (Mikolajewicz and Maier-Reimer 1990; Griffies and Tziperman 1995). Last, Sirkes and Tziperman (2001) point at the possibility of nonnormal growth in which a disturbance of a steady state, which in itself is linearly stable, may grow initially due to nonlinear interactions of eigenmodes (e.g., Farrell and Moore 1992). Other techniques have to be applied to detect nonnormal modes of variability.

Admittedly, the model configuration presented here needs improvements and inclusion of many physical processes to yield a reliable representation of the global ocean circulation. Arguably one of the major deficiencies of the results presented here may be that they were produced in absence of a convective adjustment procedure. Although parameterizing the effects of smallscale convection may be of quantitative importance for the strength of the overturning and the temperatures in the deep ocean, it is not essential for generating thermohaline circulation (Marotzke et al. 1988; Marotzke and Scott 1999). Its inclusion may have resulted in an increase of the basic-state overturning strength in this model, and it may have reduced the periods of the oscillations to some extent because of a more efficient 
exchange between the deep and shallow layers in the subpolar basins. Yet, the existence of the overturning modes presented here is likely to be robust. One may argue that the absence of convective adjustment excludes the possibility that deep-decoupling oscillations are found. Dynamically, these deep-decoupling oscillations differ from overturning oscillations in that the latter arise through a Hopf bifurcation that destabilizes a steady state ("soft" loss of stability), whereas the former is characterized by a global bifurcation in which the steady state ceases to exist and the oscillation immediately acquires a finite amplitude ("hard" loss of stability, Cessi 1996). Hence, deep-decoupling oscillations can not be found as eigenmodes of the system.

Although this model has some obvious shortcomings, we think that the results presented in this paper show that the combination of advanced numerical methods and dynamical systems theory may provide powerful tools that complement the common ocean modeling practice in unraveling the complex nonlinear dynamics of the ocean circulation. The identification of the oscillatory modes may be considered as a first step toward a complete and systematic description of the ultralow frequency variability of the global ocean circulation. Indeed, oscillations like the ones found here may be underlying the variability that appears to pervade the present Holocene period, giving rise to global climate events like the Medieval Warm Period and the Little Ice Age (Bond et al. 1997). Geochemical observations suggest that these oscillations are indeed accompanied by reorganizations in the deep ocean circulation (Broecker 2000). Furthermore, these linear modes may evolve into large-amplitude oscillations once they are properly excited (Sakai and Peltier 1996). It is therefore not impossible that similar modes lie at the heart of the Dansgaard-Oeschger cycles.

The answer to the question of whether these modes really play a role in shaping climate variability on millennial timescales must be sought in paleoceanographic data. Especially phase relationships between distinct parts of the global ocean may provide the necessary constraints to identify the oscillation responsible for a certain mode of variability. As an example, our oscillatory modes 1 and 3 display a distinctive phase relationship between the North Atlantic and Pacific Oceans (Fig. 10). This would leave a unique imprint in the sedimentary record that could be used to identify the mode responsible for the variability. With the steadily increasing number and quality of sediment cores, proxies, and dating techniques, establishing such phase relationships between ocean basins on millennial timescales seems to be within reach. Indeed, Kiefer et al. (2001) found strong evidence for an antiphase relationship between the North Atlantic and the North Pacific sea surface temperatures during Dansgaard-Oeschger events, as displayed by mode 3 in Fig. 10. Time will tell whether such interocean correlations can be estab-

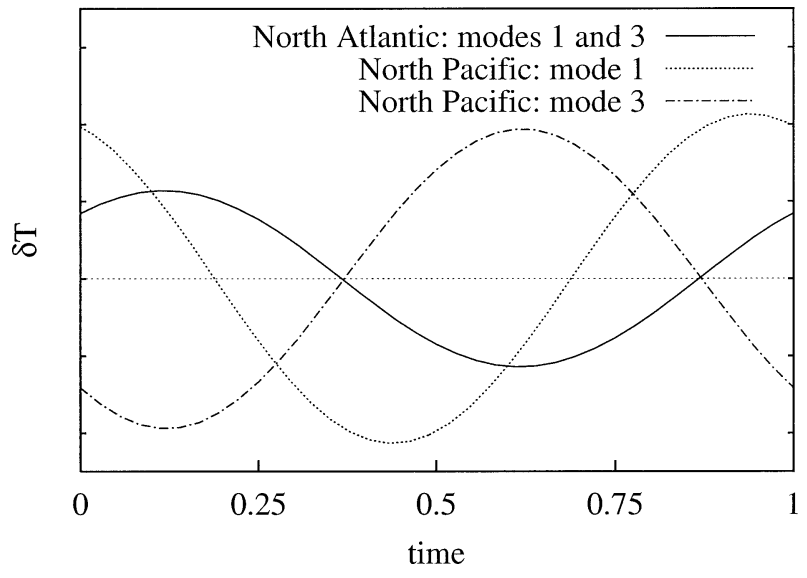

FIG. 10. Temperature anomalies at $900-\mathrm{m}$ depth in the North Atlantic and North Pacific Oceans for one oscillatory cycle of modes 1 and 3. Time is scaled with the period of each oscillation; the amplitudes of the anomalies are undetermined, but the amplitudes are mutually consistent. Note that the Pacific and Atlantic signals in mode 3 are in perfect antiphase, whereas in mode 1 they are almost $1 / 4$ out of phase.

lished for variability with much smaller amplitude as well.

Acknowledgments. This work was supported by the Netherlands Organization for Scientific Research (N.W.O.) under a PIONIER grant to HAD. We appreciate a fruitful discussion with Fei-Fei Jin (SOEST, Hawaii).

\section{APPENDIX}

\section{Temporal Evolution of Eigenmodes}

Suppose that we perturb the steady state at $t=0$, and that the perturbation has a small amplitude and the spatial structure of $\mathbf{u}_{r}$, which is the real part of a complex conjugate pair of eigenvectors $\mathbf{u}=\mathbf{u}_{r}+i \mathbf{u}_{i}$. Then the time evolution of the thermal anomaly $T^{\prime}(\mathbf{x}, t)$ is given by

$$
\begin{aligned}
T^{\prime}=\left(T_{r}\right. & \left.+i T_{i}\right) e^{\left(\varsigma_{r}+i \varsigma_{i}\right) t} \\
=e^{\varsigma_{r} t} & {\left[\left(T_{r} \cos \varsigma_{i} t-T_{i} \sin \varsigma_{i} T\right)\right.} \\
& \left.\quad+i\left(T_{r} \sin \varsigma_{i} t+T_{i} \cos \varsigma_{i} t\right)\right],
\end{aligned}
$$

and its time derivative is given by

$$
\begin{aligned}
\frac{\partial T^{\prime}}{\partial t}=e^{\varsigma_{r} t}\left[T_{r}\left(\varsigma_{r} \cos \varsigma_{i} t-\varsigma_{i} \sin \varsigma_{i} t\right)\right. \\
\left.-T_{i}\left(\varsigma_{r} \sin \varsigma_{i} t+\varsigma_{i} \cos \varsigma_{i} t\right)\right] \\
+i e^{\varsigma_{r} t}\left[T_{r}\left(\varsigma_{r} \sin \varsigma_{i} t+\varsigma_{i} \cos \varsigma_{i} t\right)\right. \\
\left.+T_{i}\left(\varsigma_{r} \cos \varsigma_{i} t-\varsigma_{i} \sin \varsigma_{i} t\right)\right] .
\end{aligned}
$$

Dynamically, this time derivative satisfies 


$$
\frac{\partial T^{\prime}}{\partial t}=\mathcal{F}_{1}+\mathcal{F}_{2}+\kappa_{h} \nabla_{h}^{2} T^{\prime}+\kappa_{v} \frac{\partial^{2} T^{\prime}}{\partial z^{2}}
$$

with

$$
\mathcal{F}_{1}=-\mu_{1} \overline{\mathbf{u}} \cdot \boldsymbol{\nabla} T^{\prime} \text { and } \mathcal{F}_{2}=-\mu_{2} \mathbf{u}^{\prime} \cdot \nabla \bar{T},
$$

where the steady solution is denoted by an overbar and the anomalies by a prime and where $\mu_{1}=\mu_{2}=1$. For convenience, the interaction between the background flow and thermal (and saline) anomalies will be denoted as $\mathcal{F}_{1}$, whereas the interaction between the flow anomalies and the background thermal (and saline) stratification as $\mathcal{F}_{2}$. Inserting Eq. (A1) in the right-hand side of Eq. (A3), equating Eqs. (A3) and (A2), and taking the sines and cosines together, yields the following expressions:

$$
\begin{aligned}
\varsigma_{r} T_{r}-\varsigma_{i} T_{i}= & -\mu_{i} \overline{\mathbf{u}} \cdot \nabla T_{r}-\mu_{2} \mathbf{u}_{r} \cdot \nabla \bar{T} \\
& +\kappa_{h} \nabla_{h}^{2} T_{r}+\kappa_{v} \frac{\partial^{2} T_{r}}{\partial z^{2}} \text { and } \\
\varsigma_{r} T_{i}+\varsigma_{i} T_{r}= & -\mu_{1} \overline{\mathbf{u}} \cdot \nabla T_{i}-\mu_{2} \mathbf{u}_{i} \cdot \nabla \bar{T} \\
& +\kappa_{h} \nabla_{h}^{2} T_{i}+\kappa_{v} \frac{\partial^{2} T_{i}}{\partial z^{2}} .
\end{aligned}
$$

Eliminating $\boldsymbol{s}_{i}$ or $\boldsymbol{s}_{r}$ from these equations, taking the volume integral (denoted by $\langle\cdot\rangle$ ), and inserting the boundary conditions, yields

$$
\begin{aligned}
\boldsymbol{\varsigma}_{r}=\frac{1}{\left\langle T_{r}^{2}+T_{i}^{2}\right\rangle}[ & \mu_{2}\left\langle\bar{T}\left(\mathbf{u}_{r} \cdot \boldsymbol{\nabla} T_{r}+\mathbf{u}_{i} \cdot \boldsymbol{\nabla} T_{i}\right)\right\rangle \\
& -\kappa_{h}\left\langle\left(\nabla_{h} T_{r}\right)^{2}+\left(\nabla_{h} T_{i}\right)^{2}\right\rangle \\
& \left.-\kappa_{v}\left\langle\left(\frac{\partial T_{r}}{\partial z}\right)^{2}+\left(\frac{\partial T_{i}}{\partial z}\right)^{2}\right\rangle\right]
\end{aligned}
$$

and

$$
\begin{aligned}
\varsigma_{i}=\frac{1}{\left\langle T_{r}^{2}+T_{i}^{2}\right\rangle}[ & \mu_{1}\left\langle\overline{\mathbf{u}} \cdot\left(T_{i} \nabla T_{r}-T_{r} \boldsymbol{\nabla} T_{i}\right)\right\rangle \\
& \left.-\mu_{2}\left\langle\bar{T}\left(\mathbf{u}_{r} \cdot \nabla T_{i}-\mathbf{u}_{i} \cdot \nabla T_{r}\right)\right\rangle\right] .
\end{aligned}
$$

These expressions show how the growth rate and oscillation period depend on the several feedback parameters and on correlations between the spatial patterns of the eigenmodes and the basic state. In particular, the growth rate directly depends on $\mathcal{F}_{2}$ and on vertical and horizontal mixing, whereas the period directly depends on the advective feedbacks only.

\section{REFERENCES}

Alley, R. B., S. Anandakrishnan, and P. Jung, 2001: Stochastic resonance in the North Atlantic. Paleoceanography, 16, 190-198.

Bond, G., and Coauthors, 1997: A pervasive millenial-scale cycle in North Atlantic Holocene and glacial climates. Science, 278, $1257-1266$.
- , and Coauthors, 2001: Persistent solar influence on North Atlantic climate during the Holocene. Science, 294, 2130-2136.

Broecker, W. S., 2000: Abrupt climate change: Causal constraints provided by the paleoclimate record. Earth-Sci. Rev., 51, 137154.

— D. M. Peteet, and D. Rind, 1985: Does the ocean-atmosphere system have more than one stable mode of operation? Nature, 315, 21-26.

, and Coauthors, 1998: How much deep water is formed in the Southern Ocean? J. Geophys. Res., 103, 15 833-15 843.

Brunet, G., 1994: Empirical normal-mode analysis of atmospheric data. J. Atmos. Sci., 51, 932-952.

Cessi, P., 1996: Convective adjustment and thermohaline excitability. J. Phys. Oceanogr., 26, 481-491.

Dewar, W. K., and R. X. Huang, 1995: Fluid flow in loops driven by freshwater and heat fluxes. J. Fluid Mech., 297, 153-191.

Dijkstra, H. A., 2000: Nonlinear Physical Oceanography. Kluwer Academic, $456 \mathrm{pp}$.

— , and M. J. Molemaker, 1997: Symmetry breaking and overturning oscillations in thermohaline-driven flows. J. Fluid Mech., 331, 195-232.

— , H. Öksüzoglu, F. W. Wubs, and E. F. F. Botta, 2001: A fully implicit model of the three-dimensional thermohaline ocean circulation. J. Comput. Phys., 173, 685-715.

Farrell, B. F., and A. M. Moore, 1992: An adjoint method for obtaining the most rapidly growing perturbation to oceanic flows. J. Phys. Oceanogr., 22, 338-349.

Ganopolski, A., and S. Rahmstorf, 2002: Abrupt glacial climate changes due to stochastic resonance. Phys. Rev. Lett., 88, doi: 10.1103/PhysRevLett.88.038501.

,$- \ldots$, V. Petoukhov, and M. Claussen, 2001: Rapid changes of glacial climate simulated in a coupled climate model. Nature, 409, 153-158.

Griffies, S. M., and E. Tziperman, 1995: A linear thermohaline oscillator driven by stochastic atmospheric forcing. J. Climate, $\mathbf{8}$, $2440-2453$.

Huck, T., and G. K. Vallis, 2001: Linear stability analysis of the three-dimensional thermally-driven ocean circulation: Application to interdecadal oscillations. Tellus, 53A, 526-545.

Keller, H. B., 1977: Numerical solution of bifurcation and nonlinear eigenvalue problems. Applications of Bifurcation Theory, P. H. Rabinowitz, Ed., Academic Press, 359-384.

Kiefer, T., M. Sarntheim, H. Erlenkeuser, P. M. Grootes, and A. P. Roberts, 2001: North Pacific response to millennial-scale changes in ocean circulation over the last 60 kyr. Paleoceanography, 16, 179-189.

Levitus, S., and T. Boyer, 1994: Temperature. Vol. 4, World Ocean Atlas 1994, NOAA Atlas NESDIS 4, 117 pp.

_- R. Burgett, and T. Boyer, 1994: Salinity. Vol. 3, World Ocean Atlas 1994, NOAA Atlas NESDIS 3, 99 pp.

Marotzke, J., 1989: Instabilities and multiple steady states of the thermohaline circulation. Oceanic Circulation Models: Combining Data and Dynamics, D. L. T. Anderson and J. Willebrand, Eds., NATO ASI Series, Vol. 284, Springer-Verlag, 501-511.

—_, and J. R. Scott, 1999: Convective mixing and the thermohaline circulation. J. Phys. Oceanogr., 29, 2962-2970.

__ P. Welander, and J. Willebrand, 1988: Instability and multiple steady states in a meridional-plane model of thermohaline circulation. Tellus, 40, 162-172.

Mayewski, P. A., L. D. Meeker, M. S. Twickler, S. Whitlow, Q. Yang, W. B. Lyons, and M. Prentice, 1997: Major features and forcing of high-latitude Northern Hemisphere atmospheric circulation using a 110,000-year-long glaciochemical series. J. Geophys. Res., 102, 26 345-26 366.

Mikolajewicz, U., and E. Maier-Reimer, 1990: Internal secular variability in an ocean general circulation model. Climate Dyn., 4, 145-156.

Mysak, L., T. F. Stocker, and F. Huang, 1993: Century-scale variability in a randomly forced, two-dimensional thermohaline ocean circulation model. Climate Dyn., 8, 103-116. 
Nowlin, W. D., and J. M. Klinck, 1986: The physics of the Antarctic Circumpolar Current. Rev. Geophys., 24, 469-491.

Oppo, D. W., J. F. McManus, and J. L. Cullen, 1998: Abrupt climate events 500,000 to 340,000 years ago: Evidence from subpolar North Atlantic sediments. Science, 279, 1335-1338.

Osborn, T., 1997: Thermohaline oscillations in the LSG OGCM: Propagating anomalies and sensitivity to parameterizations. J. Phys. Oceanogr., 27, 2233-2255.

Pedlosky, J., 1987: Geophysical Fluid Dynamics. Springer-Verlag, $710 \mathrm{pp}$.

Pierce, D. W., T. P. Barnett, and U. Mikolajewicz, 1995: Competing roles of heat and freshwater flux in forcing thermohaline circulation. J. Phys. Oceanogr., 25, 2046-2064.

Quon, C., and M. Ghil, 1995: Multiple equilibria and stable oscillations in thermosolutal convection at small aspect ratio. J. Fluid Mech., 291, 33-56.

Sakai, K., and W. R. Peltier, 1995: A simple model of the Atlantic thermohaline circulation: Internal and forced variability with paleoclimatological implications. J. Geophys. Res., 100, 13 45513479 .

—, and $-1996:$ A multibasin reduced model of the global thermohaline circulation: Paleoceanographic analyses of the origins of ice-age climate variability. J. Geophys. Res., 101, 22 535-22 262.

— and _ 1997: Dansgaard-Oeschger oscillations in a coupled atmosphere-ocean climate model. J. Climate, 10, 949-970.

$\longrightarrow$, and — 1999: A dynamical systems model of the DansgaardOeschger oscillation and the origin of the Bond cycle. J. Climate, 12, 2238-2255.

Schmidt, G. A., and L. A. Mysak, 1996: The stability of a zonally averaged thermohaline circulation model. Tellus, 48A, 158-178.

Scott, J., J. Marotzke, and P. H. Stone, 1999: Interhemispheric thermohaline circulation in a coupled box model. J. Phys. Oceanogr., 29, 351-365.

Sirkes, Z., and E. Tziperman, 2001: Identifying a damped oscillatory thermohaline mode in a general circulation model using an adjoint method. J. Phys. Oceanogr., 31, 2297-2306.

Sleijpen, G. L. G., and H. A. Van der Vorst, 1996: A Jacobi-Davidson iteration method for linear eigenvalue problems. SIAM J. Matrix Anal. Appl., 17, 410-425.

Stommel, H., 1961: Thermohaline convection with two stable regimes of flow. Tellus, 13, 244-230.

Te Raa, L. A., and H. A. Dijkstra, 2002: Instability of the thermohaline ocean circulation on interdecadal timescales. J. Phys. Oceanogr., 32, 138-160.

Titz, S., T. Kuhlbrodt, S. Rahmstorf, and U. Feudel, 2002: On freshwater-dependent bifurcations in box models of the interhemispheric thermohaline circulation. Tellus, 54A, 89-98.

Trenberth, K. E., J. G. Olson, and W. G. Large, 1989: A global ocean wind stress climatology based on ECMWF analyses. National Center for Atmospheric Research Tech. Note NCAR/ TN-338+STR, Boulder, CO, 93 pp.

Tziperman, E., J. R. Toggweiler, Y. Feliks, and K. Bryan, 1994: Instability of the thermohaline circulation with respect to mixed boundary conditions: Is it really a problem for realistic models? J. Phys. Oceanogr., 24, 217-232.

Vellinga, M., 1996: Instability of two-dimensional thermohaline circulation. J. Phys. Oceanogr., 26, 305-319.

Weaver, A. J., and T. M. Hughes, 1994: Rapid interglacial climate fluctuations driven by North Atlantic ocean circulation. Nature, 367, 447-450.

Weijer, W., and H. A. Dijkstra, 2001: A bifurcation study of the threedimensional thermohaline ocean circulation: The double hemispheric case. J. Mar. Res., 59, 599-631.

_ _ _ - H. Öksüzoglu, F. W. Wubs, and A. C. De Niet, 2003: A fully-implicit model of the global ocean circulation. J. Comput. Phys., in press.

Welander, P., 1982: A simple heat-salt oscillator. Dyn. Atmos. Oceans, 6, 233-242.

_ 1 1986: Thermohaline effects in the ocean circulation and related simple models. Large Scale Transport Processes in Oceans and Atmosphere, J. Willebrand and D. L. T. Anderson, Eds., D. Reidel, 163-200.

Winton, M., and E. S. Sarachik, 1993: Thermohaline oscillations induced by strong steady salinity forcing of ocean general circulation models. J. Phys. Oceanogr., 23, 1389-1410. 